\section{PKDB - the International Paleoclimate Database}

The International Paleoclimate Database (PKDB) is part of the research program "Terrestrial Paleoclimatology" of the German Federal Government. Both, the database and the program, are headed by Prof. Dr. Dr. h.c. B. Frenzel.

PKDB is a multi-proxy database dedicated to the storage of climatological and ecological data of the whole earth for the Quaternary and part of the Tertiary. Developing a comprehensive database for all the very heterogeneous data sources that have to be taken into consideration is the great scientific and technological challenge for PKDB.

Focal points of our work are:

1. Extraction of relevant data from literature

2. Longtime archiving of data produced by the projects of the "Terrestrial

Paleoclimatology" and other similar programs

3. Integration of public data from related data centers

4. Critical analysis of the stored data

5. The reconstruction of the global climate and ecology for the Quaternary

6. Maintenance of the archive with up-todate data

7. Free and convenient access to the holdings of the database

8. Compatibility with other paleodata efforts such as the WDC-A in Boulder, and in Germany with CERA and PANGAEA

This work is at the moment done by five scientists at the Botanical Institute of the Hohenheim University, Stuttgart.

\section{Structure and Contents of the Database}

The database is based on a relational database system (EMPRESS) and is composed of:

- a large bibliographic table (> 300,000 references)

- data tables: META-data $(>70,000)$ with corresponding tables for header data, tabular data, datings, soil data

- climate data tables for present-day and reconstructed values (spot and grid data)

Additional tables exists for taxonomy, excerpts and abstracts, site descriptions, stratigraphic correlations, images, climate indicators etc.

Both, original data and data added by the critical analysis, are stored and their origin is clearly indicated. All data can be updated, when, for example, progress in dating technology is made.

\section{WWW-Interface}

Public access to the holdings of PKDB started with a 4GL-Interface for VT220-terminals and X-Windows. From August 1997 on we began to offer access to the PKDB tables by a publicly available WWW interface.

The data holdings can be accessed from two query forms:

The first form is for a bibliographical query through the tables for bibliography, excerpts and abstracts. The results can be obtained as a table or as a reference list (figure below). Any available data associated with a given reference is indicated by the presence of a data icon. The data itself is then accessible by clicking on this icon.

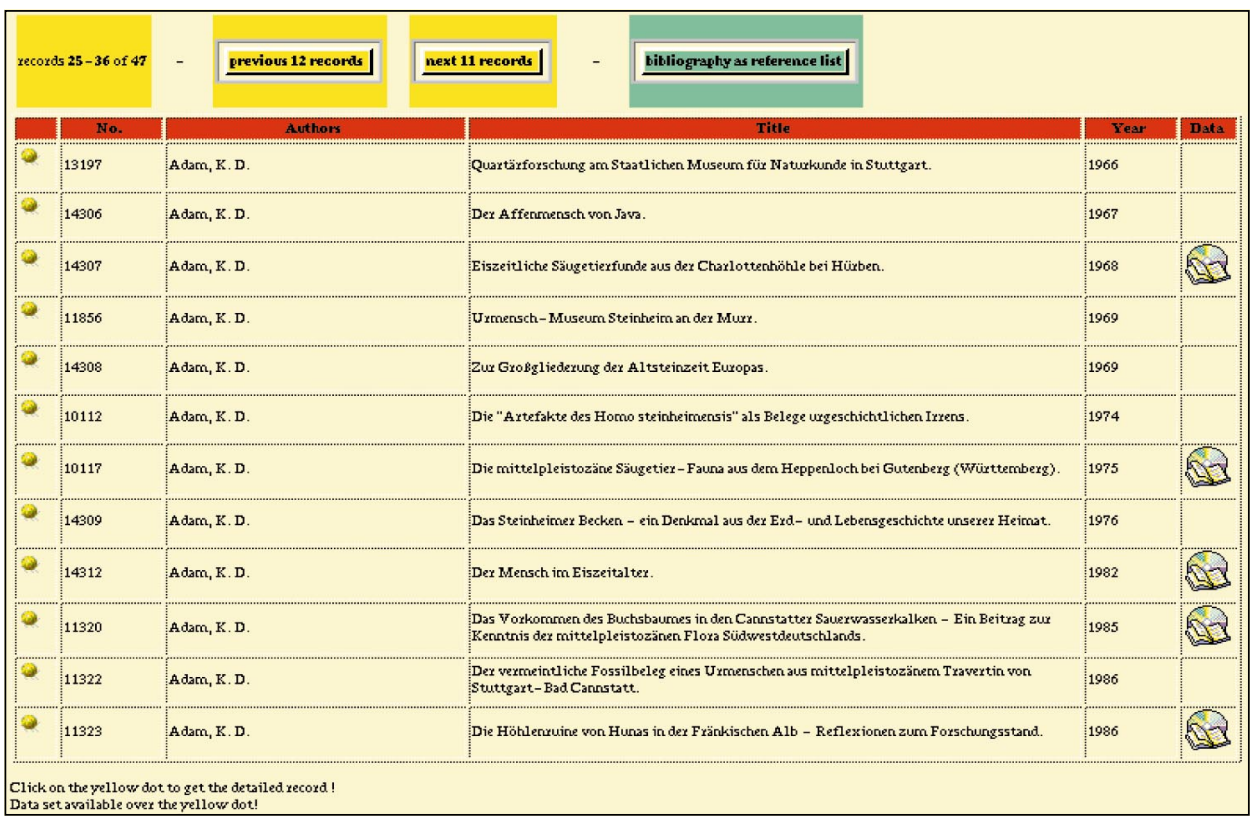

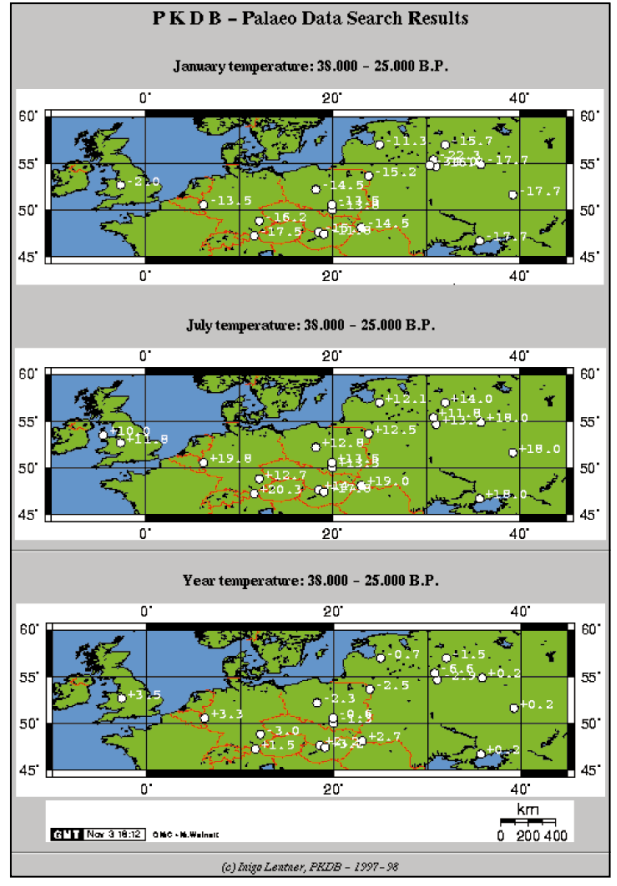

Climate reconstruction maps produced with the web-interface from a data query

The second form is for a query through the data tables, with the possibility of restricting the query by data group, coordinates, altitude or time period. The result is shown in a overview table, from which the detailed data sets can be accessed with a further click. The geographical distribution of the data is shown on a variety of maps.

The next release of this query program, which is now in a test phase, will allow additional qualifications on data queries, different output formats, and even queries for tables and maps of taxonomic distribution and some climate reconstruction time slices (figure above). Ideas for further improvements are numerous.

\section{Outlook}

Negotiations are under way for the longrange safeguard of the database under the auspices of the German "Program of Academies". This will allow us to accomplish our goals, not only to be an archive for paleoclimatic data, but also to maintain the contents of the database up-to-date as the science progresses.

\section{INIGO LENTNER}

PKDB, Institut für Botanik, Universität Hohenheim, Stuttgart, Germany

lentner@pkdb.botanik.uni-hohenheim.de http://www.pkdb.uni-hohenheim.de

Reference table produced with the web-interface from a bibliographic query 